\title{
Analysis of size distributions of type II ZnTe/ZnSe quantum dots
}

\author{
Johnson Lee ${ }^{1}$, Chu Shou Yang ${ }^{2}$, Chun Tai Chang ${ }^{1}$, Jerron Liu', Wu Ching Chou, ${ }^{*}$, \\ Chih Ming Lai ${ }^{3}$, Gwo Jen Jan ${ }^{4}$, and Yin Sheng Huang ${ }^{5}$ \\ ${ }^{1}$ Department of Physics, Chung Yuan Christian University, Chung-Li, Taiwan 32023, R.O.C. \\ 2 Department of Electrophysics, National Chiao Tung University, Hsin-Chu, Taiwan 30010, R.O.C. \\ ${ }^{3}$ Department of Electronic Engineering, Ming Chuan University, Taoyuan, Taiwan 333, R.O.C. \\ ${ }^{4}$ Department of Electrical Engineering, National Taiwan University, Taipei, Taiwan 10617, R.O.C. \\ 5 Department of Electronic Engineering, National Taiwan University of Science and Technology, \\ Taipei, Taiwan 10617, R.O.C.
}

Received 1 March 2004, revised 19 September 2004, accepted 23 September 2004

Published online 8 November 2004

\section{PACS 68.65.Hb, 68.37.Ps, 78.55.Et, 78.67.Hc}

The photoluminescence (PL) spectrum of the $\mathrm{ZnTe} / \mathrm{ZnSe}$ quantum dot (QD) structure which has a type II band alignment was investigated. A broader structure peaking at $2.180 \mathrm{eV}$ together with the features of the $\mathrm{ZnSe}$ buffer layer located at $2.820 \mathrm{eV}$ in the PL spectrum at $10 \mathrm{~K}$ were observed. The broadness of the PL attributes to the spatial inhomogeneity of the QD sizes. The PL spectrum was a normal distribution with a variance of $0.002 \mathrm{eV}^{2}$. However, the dot size distribution was a deformed Gaussian. Using only one set of data of dot size distribution measured by the Atomic Force Microscopy (AFM), the statistical estimates with standard errors were evaluated using the bootstrap methodology. The probability distribution was inferred by calculating the means, percentiles and correlation coefficients of the base diameter and the height of the QDs. We concluded that the dot distributions were indeed a deformed Gaussian and the correlation coefficient of the diameter and the height of the QDs was $0.49 \pm 0.01$. The uniformity of the dot size distribution was poor. To infer the dot size distribution from the PL spectrum, we numerically solved the Schrödinger equation by elaborating on the orthogonal periodic functions (OPF) approximation for the type II lens shaped QDs. The convergence of the probability density was discussed in a great detail. We found that the band edge discontinuities inside the dot acts as a barrier with $\Delta E_{c}=315 \mathrm{meV}$ for the conduction band and as a well with $\Delta E_{v}=735 \mathrm{meV}$ for the valence band. In the AFM measurement, the offset in dot height is $14 \AA$ and the aspect ratio is 0.04 . We employed the Schrödinger equation to correlated the PL spectrum (intensity versus optical energy) to the AFM data (number of QDs versus dot size) and the agreement was excellent.

\section{Introduction}

Recent advances in semiconductor technology such as molecular beam epitaxy (MBE) together with very accurate lithographic techniques made it possible to fabricate quasi-one-dimensional semiconductor structures to confine electrons known as the quantum wire [1,2]. Reed et al. [3] achieved complete quantization of free electron motion by trapping electrons in a quasi-zero-dimensional semiconductor quantum dot (QD). Using high strain epitaxy technique for the production of quasi-zero-dimensional QDs, the Stranski-Krastanov $(\mathrm{S}-\mathrm{K})$ growth method $[4,5]$ is the most popular. The semiconductor QDs formed in the $\mathrm{S}-\mathrm{K}$ phase transition are called self-organized or self-assembled dots (SADs). Using various crystal growth methods and conditions, different dot shapes have been grown and reported. Dots in the shape of

\footnotetext{
" Corresponding author: e-mail: wuchingchou@mail.nctu.edu.tw
} 
pyramids [6], cylinders or disks $[2,7,8]$, cones [9] and lenses [4, 10] have been studied, although actual determinations of the shapes have not been definite. Using MBE, GaN QDs in an AIN matrix were successfully grown on $\mathrm{Si}$ (111) by the molecular beam epitaxy [11]. Stacking of QD planes with properly chosen dot sizes were demonstrated to emit white light. Furthermore, light-emitting diodes made from the II-VI compound semiconductor QDs were reported [12]. Although a large amount of effort placed on the fabrication of opto-electronic devices based on QDs, the focus of interest remains on the novel physical properties exhibited in the material systems of QDs. Time evolution of dot shapes during and after growth remains a problem $[13,14]$. Neither the growth dynamics nor the phase diagram of the whole II-VI compound semiconductor family is fully understood. Every real ensemble of nanocrystals dispersed in some environment possesses unavoidable inhomogeneities and for the majority of matrices, this is the size distribution. The photoluminescence (PL) spectra of $\mathrm{ZnSe} / \mathrm{ZnS}$ QDs with $[15,16]$ or without [17] cap layers were investigated to reveal QD size distributions. In order to correlate the dot size with the photon energy of the PL spectrum, Tawara et al. [17] adopted an infinite well model for quantum boxes to estimate the quantum confinement sub-band energies by solving the Schrödinger equation. A histogram of the size distribution was obtained from a PL spectrum by mapping the intensity into the number of QDs measured by the Atomic Force Microscopy (AFM). Lee et al. [18] investigated the PL spectra of ZnTe/ZnSe QDs to correlate the dot size with the photon energy by adopting a finite well model for cylindrical QDs by solving the Schrödinger equation together with the first-order perturbation correction. Unfortunately, in reference [18], only the theoretical predictions were presented without AFM measurements.

The theoretical calculations of the basic properties of the two-dimensional electron or hole systems in quantum wells were well developed. However, the geometry of SADs with additional degrees of quantum confinement (due to the finite potential barriers) imposes boundary conditions that complicate the problems in solving the Schrödinger equation. If the shape of SADs is spherical, the solutions can be obtained analytically [19]; otherwise, numerical methods [20, 21] must be applied. Gangopadhyay et al. [19] suggested a numerical method by extending the orthogonal periodic functions (OPF) to effectively evaluate the eigenenergies of SADs with a parallelopiped shape. Califano et al. [22-24] extended this method to study pyramidal, cuboidal, and cubic SADs. In order to calculate energy levels of pyramidal shaped InAs/GaAs dots, a $6859 \times 6859$ matrix was solved and was the minimum number required to achieve convergence for the eigenvalues to within less than $1 \mathrm{meV}$ [24]. Wojs et al. [25] focused on lens-shaped SAD with the adiabatic approximation. When the valence band mixing and the spatial variation of the confining potentials are significant due to strain, the confinement energy levels must be evaluated by using those more sophisticated models [26-29] such as an $8 \times 8$ Hamiltonian from the eight-band k.p theory.

It is well known that since semiconductor lasers are often heavily doped and operate at high injected carrier densities, it is necessary to consider the effects of these high doping densities on the density of states, the energy band profiles, and the binding energies. Depending on the doping profiles of the semiconductors, many-particle interactions can cause band bendings and deform the energy band profiles. For the QD lasers with type II materials, the holes may reside inside the dots while the electrons may reside in the matrix materials. Due to the screened Coulomb interaction between the electrons and holes, a band bending effect can occur. The screening effect plays an important role in the calculation of the optical gain for the strained $\mathrm{GaAsSb} / \mathrm{GaAs}$ quantum-well lasers [30].

The main purpose of this paper is to report the results of our investigation on the size distributions of type II ZnTe QDs on a ZnSe buffer layer. This paper is an extension of reference [18]. Important parameters of the QD distributions were inferred by simulating PL spectra. The means, the percentiles and the correlation coefficients of the dot sizes were estimated with the Bootstrap methodology [31, 32]. A brief discussion of the bootstrap is in section 4. In section 2, the growth of the ZnTe/ZnSe QDs and the PL measurements are described. In section 3, main steps of how to correlate the PL spectrum with the dot size distributions are presented. Section 4 presents the results of our numerical calculations and describes how to infer the dot size distributions from the PL spectra. Discussions focus on the reliability and the physical implications of the numerical results. A summary is presented in section 5. In Appendix A, the OPF approximation [19, 22-24] is further elaborated for the type I and II symmetroidal QDs and is applied to study the lens shaped QDs. 


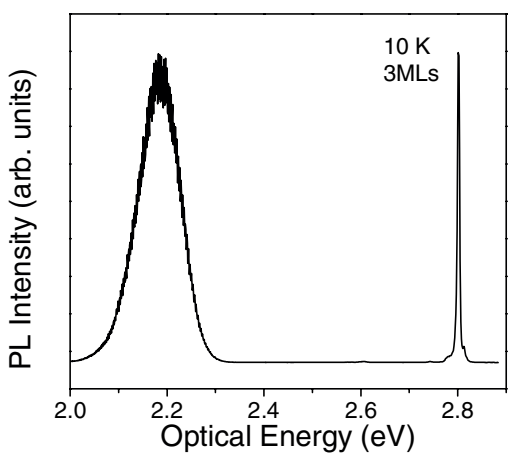

Fig. 1 Photoluminescence spectrum of the type II ZnTe/ZnSe QDs at $10 \mathrm{~K}$. The average coverage of $\mathrm{ZnTe}$ is 3 MLs.

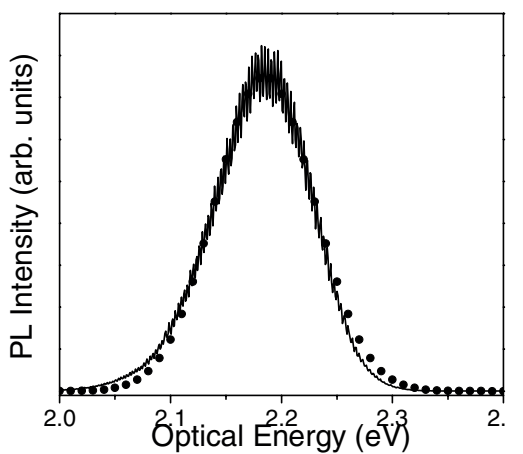

Fig. 2 High resolution photoluminescence spectrum of the type II ZnTeZnSe QDs 3 MLs sample. The vertical axis is the optical intensity $I$ in arbitrary units (a.u.). The dotted line was fitted to the Gussian curve.

\section{Experiment}

Self-assembled type II ZnTe QDs were grown on the GaAs substrate with a ZnSe buffer layer by MBE. The ZnSe buffer layer includes several MLs grown by the migration enhance epitaxy (MEE) [33] and a thickness of $20 \mathrm{~nm}$ grown by the conventional MBE growth mode. The average roughness of the $\mathrm{ZnSe}$ buffer layer is about $0.28 \mathrm{~nm}$. Followed the deposition of flat $\mathrm{ZnSe}$ buffer layer, the growth of selfassembled ZnTe QDs started. The average coverage of ZnTe for samples was 3 mono-layers (MLs). For the PL study, a ZnSe cap layer of $50 \mathrm{~nm}$ was grown. The $351 \mathrm{~nm}$ line of an Argon ion laser was used to excite the PL spectra and a SPEX 1403 double-grating spectrometer was used to analyze the PL spectra. Slit widths were set to $100 \mu \mathrm{m}$ to yield a spectrum resolution better than $0.1 \mathrm{meV}$.

We begin our discussion with a PL spectrum of a 3 MLs ZnTe QD at $10 \mathrm{~K}$ as shown in Fig. 1. A near band edge emission, free and impurity bound excitons, of $\mathrm{ZnSe}$ buffer layer was observed at $2.820 \mathrm{eV}$. A broader PL structure peaking at $2.180 \mathrm{eV}$ was observed and enlarged with a higher resolution as shown in Fig. 2. The dotted curve shown in Fig. 2 was obtained by fitting the PL line shape with a Gaussian curve and is discussed in section 4 . The broadness of the PL implies the spatial inhomogeneity of the QD sizes $[34,35]$. To directly investigate the spatial inhomogeneity, the height and diameter measurement was carried out immediately after the growth without a cap layer on top of the ZnTe QDs. The NT-MDT SOLVER P47 AFM was used for the morphology study and the measurement was carried out using the semi-contact mode. The scan step in the $x, y$ directions were both $4.8 \mathrm{~nm}$ and the resolution in the $z$-direction is $0.01 \mathrm{~nm}$. The shape of silicon tip is conic. The diameter and height of the tip were 30 and $70 \mathrm{~nm}$ (i.e. the aspect ratio, $\alpha=\mathrm{H} / \mathrm{D}=7 / 3$ ), respectively. The aspect ratio of silicon tip is much lager than that of the ZnTe QDs grown in current study. The measured dot size distribution, $N_{\mathrm{QD}}$, is presented in Fig. 3 and discussed in Section 4.

\section{PL spectrum and dot size distribution}

We realized that the broadness of the PL comes from the spatial inhomogeneity of the QD sizes and that the dot size distribution and the photon energy of the PL spectrum must be correlated. In our experiment, since all samples are undoped and the optical pumping power is low, the screening effect due to the many-particle interactions is neglected in this paper. We estimate the quantum confinement sub-band energies of the type II QDs by elaborating the well-known OPF approximation [19, 22-24] as described in Appendix A for reference. By substituting Eq. (A16) into Eqs. (A12)-(A15) together with the matrix elements Eq. (A17) in Appendix A, the quantum confinement energies and corresponding wavefunctions for the type II materials with lens shaped QDs were obtained. 


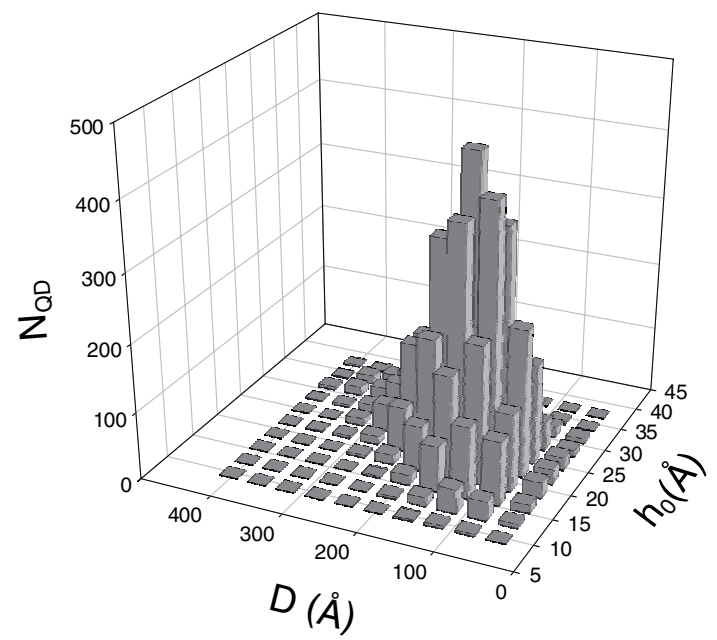

Fig. 3 (online colour at: www.pss-b.com) Dot size distribution: number of QDs $N_{\mathrm{QD}}$ versus base diameter $D$ and height $h_{0}$ in $\AA$ measured by AFM. There are 5187 dots in an area of $3 \times 3 \mu^{2}$.

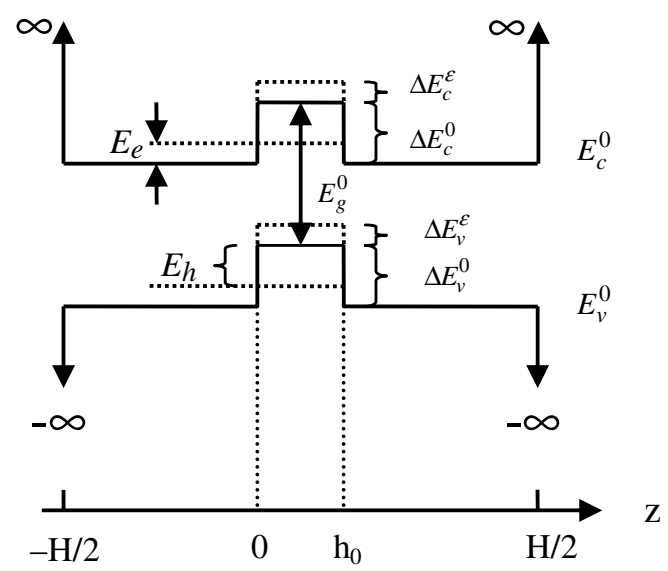

Fig. 4 Schematics of the band alignment of the type II ZnTe/ZnSe QDs along the $z$-axis at $r=0$.

A schematic of the band alignment of the type II ZnTe/ZnSe QD along the $z$-axis with $r=0$ is plotted for the conduction (valence) band edge, $E_{c}\left(E_{v}\right)$, in Fig. 4. Referring to Fig. A2 for the geometry of a lens, the lens height, $h_{\text {lens }}$, varies with $r$ (see Eq. (A16)). For $r=0$, we have $z=h_{0}$, where $\mathrm{h}_{0}$ is the maximum height of the lens. Note that because of the lattice mismatch, the system is highly strained.

Figure 4 shows that for the type II ZnTe/ZnSe structure inside the QD region $\left(0 \leq z \leq h_{0}\right)$, the conduction electrons see a barrier with a barrier height of $\Delta E_{c}^{0}+\Delta E_{c}^{\varepsilon}$ for the conduction band while the heavy holes see a well with a well height of $\Delta E_{v}^{0}+\Delta E_{v}^{\varepsilon}$ for the valence band. $\Delta E_{c}^{0}\left(\Delta E_{v}^{0}\right)$ is the conduction (valence) band offset when the system is unstrained. Due to the strain effect, the amount of the energy shift for the conduction (valence) band edge is $\Delta E_{v}^{\varepsilon}\left(\Delta E_{v}^{\varepsilon}\right)$. In the matrix regions $\left(-\mathrm{H} / 2 \leq z\right.$ and $\left.h_{0}<z \leq \mathrm{H} / 2\right)$, the electrons see wide wells while the holes see wide barriers.

The particle in the QD can be an electron in the conduction band or a heavy-hole. Because we are interested in knowing the optical transitions, we have to evaluate both energy shifts. In the QD region, let $E_{g}^{0}=E_{c}^{0}-E_{v}^{0}$ be the energy difference of the bulk conduction band edge $\left(E_{c}^{0}\right)$ and the bulk valence band edge $\left(E_{v}^{0}\right)$, then the energy gap with strain is $E_{g}=E_{g}^{0}+\Delta E_{c}^{\varepsilon}-\Delta E_{v}^{\varepsilon}$. If $\Delta E_{c}^{\varepsilon}-\Delta E_{v}^{\varepsilon}$ is negligible, then $E_{g}$ can be approximated by $E_{g}^{0}$. In this paper, we assume that $\Delta E_{c}^{\varepsilon}$ almost completely cancels $\Delta E_{v}^{\varepsilon}$ and $E_{g} \approx E_{g}^{0}$ holds. Let $E_{e}$ and $E_{H}$ be the confinement energies for the electron and heavy-hole, respectively, as shown in Fig. 4. The corresponding envelope functions are $\Psi_{e}$ and $\Psi_{h}$. For the electron-heavy-hole transitions, the optical energy $E_{e H}$ is defined as:

$$
E_{e H}=E_{e}+E_{H}+E_{g}^{0}-\Delta E_{c}^{0} .
$$

We assume that the intensity $I$ of a PL spectrum is proportional to the total number of dots, $N_{\mathrm{QD}}$, of the same size and is given by

$$
I=C O N_{\mathrm{QD}},
$$

where $C$ is a constant and $O$ is the overlap integral related to $E_{e}$ and $E_{H}$. Note that $N_{\mathrm{QD}}$ is an implicit function of the optical energy. Since $O$ varies slowly with the dot size, it is a good approximation to assume that I is directly proportional to $N_{\mathrm{QD}}$ :

$$
I=C N_{\mathrm{QD}} \text {. }
$$

The intensity $I$ as a function of the optical energy was shown in Fig. 2 and the dot size distribution $N_{\mathrm{QD}}$ as functions of $D$ and $h_{0}$ measured by the AFM was shown in Fig. 3. Equation (3) correlates the dot size distribution to the intensity of the PL spectrum and is experimentally justified in Section 4. 


\section{Numerical results and discussion}

This section consists of two sub-sections. In sub-section 4.1, we estimate the dot size distribution and the dot size statistics to obtain input parameters for the Schrödinger equation; in sub-section 4.2 we discuss the confinement energies for the type II QDs and their correlation to the PL spectrum.

\subsection{Dot size distribution and statistics}

Since we treat the dot sizes as input parameters to the Schrödinger equation, we need to study the dot size distribution first. From the AFM observations, we conclude that the shape of the dot looks more like a lens. There are 5187 dots in an area of $9 \mu \mathrm{m}^{2}$ which leads to an average distance of $417 \AA$ between dots. In Fig. 3, the most probable $N_{\mathrm{QD}}$ value is 435 located at $D=164 \AA$ and $h_{0}=25 \AA$. Thus, it is reasonable to assume that there are no carrier communications between dots. The lens shaped QDs are characterized by a base diameter $D=2 \rho$ and a height $h_{0}$, as indicated in Fig. A2 in Appendix A. Figure 5 shows that a linear correlation between $D$ and $h_{0}$ is calculated to be $h_{0}=0.04 D+14$ in $\AA$ with a $R$-squared value of 0.24 by using the least squares estimate. $R$-squared is related to the $\chi$-squared. Set $N_{h}$ to be the total number of dots with the same height despite the magnitude of the diameter $D . N_{h}$ as a function of $h_{0}$ is plotted in Fig. 6. Clearly we see that the distribution of $N_{h}$ versus $h_{0}$ is a deformed Gaussian shape and has a peak around $25 \AA$. A deformed Gaussian implies that the variation of the energy with the dot size obtained from the Schrödinger equation is non-linear.

Note that because we had only one set of data for the size distribution measured from the AFM, there would be no formula to provide estimated standard errors if we wanted to acquire statistical estimators other than the mean. Any statistical estimator without the standard error is less meaningful. Hence we adopted the bootstrap method to estimate the standard errors. Bootstrap is a modern statistical technique; it is a computer-based method of inference that can answer many crucial statistical questions without assuming a particular functional form for the underlying probability density function. The bootstrap is a computer-based method for assigning measures of accuracy to statistical estimates [31]. Various statistics of the dot sizes with the standard deviations of the sample means were calculated with 100 bootstrap samples which were generated by randomly sampling with replacement from the data set as shown in Fig. 5. One hundred bootstraps were used to ensure the convergence of the estimates. The calculated mean of the base diameter $\langle D\rangle$ is $165 \pm 1 \AA$ while the mean of the height $\left\langle h_{0}\right\rangle$ is $24.59 \pm 0.06 \AA$. The $50 \%$ percentile (i.e. the medians) for $D$ is $159 \pm 1 \AA$ and for $h_{0}$ is $24.70 \pm 0.04 \AA$. The $99 \%$ percentile for $D$ is $333 \pm 6 \AA$ and for $h_{0}$ is $35.72 \pm 0.30 \AA$. The slight deviations of the means, the medians and the 0.99 percentiles for the $D$ and $h_{0}$ indicate that the distribution is a deformed Gaussian distribution and is consistent with the distribution as shown in Fig. 6. The sample correlation coefficient for $D$ and $h_{0}$ is

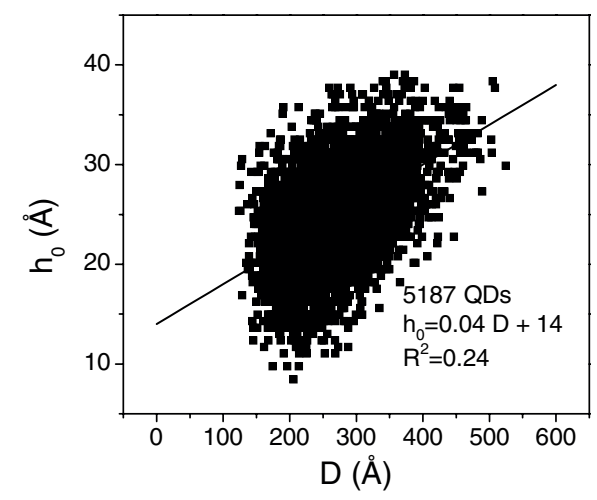

Fig. 5 Linear correlation between $h_{0}$ and $D$ in an area of $3 \times 3 \mu \mathrm{m}^{2}$.

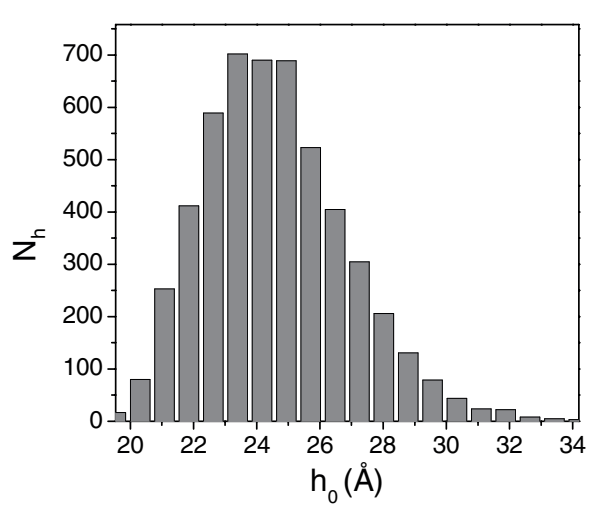

Fig. 6 Histogram of number $N_{h}$ versus $h_{0}$. $N_{h}$ is a sum of the number of dots over all possible $D$ 's with the same height $h_{0}$. 
$0.49 \pm 0.01$ which implies that when the linear relationship $h_{0}=0.04 D+14(\AA)$ as shown in Fig. 5 is used to correlate $D$ and $h_{0}$, there is a probability of 0.51 in error because of strong fluctuations of $D$ versus $h_{0}$. Finally, note that in the AFM experiment, $D$ and $h_{0}$ are measured from an arbitrary reference point on the ZnTe surface. Therefore, the linear relationship $h_{0}=0.04 D+14(\AA)$ indicates that there is an offset of $14 \AA$ in $h_{0}$ and the convolution radius of the silicon tip is excluded in $D$. After both offsets in $h_{0}$ and $D$ are excluded, the relation $h_{0}=0.04 D$ holds and the aspect ratio $\alpha=h_{0} / D=0.04$ is defined for later applications.

\subsection{Confinement energies of type II QDs and PL spectrum}

The energy levels and wavefunctions for lens shaped QDs can be numerically evaluated for the type II $\mathrm{ZnTe} / \mathrm{ZnSe}$ QDs as mentioned earlier in section 3. The eigenvalues of the matrix equation are obtained by using appropriate sub-routines in the Integrated Mathematical Statistical Library (IMSL). The low temperature band gaps of $2.820 \mathrm{eV}$ and $2.400 \mathrm{eV}$ were used for $\mathrm{ZnSe}$ and $\mathrm{ZnTe}$, respectively [36, 37]. The energy gap difference between the gap of the ZnSe and the gap of the ZnTe is $\Delta E_{g}^{0}=0.420 \mathrm{eV}$. The symbol $E_{g}^{0}$ used in Eq. (1) is the energy gap of the QD, i.e., $E_{g}^{0}=2.400 \mathrm{eV}$. For the type II ZnTe/ZnSe structure inside the dots, the conduction electrons see a barrier with a barrier height of $\Delta E_{c}^{0}=q \Delta E_{g}^{0}$ for the conduction band and the heavy holes see a well with a well height of $\Delta E_{v}^{0}=(1+q) \Delta E_{g}^{0}$ for the valence band where $q$ is a positive parameter. The following parameters $[36,37]$ are used in our calculations: $m_{0}$ for the free electron mass, $m_{e}^{*}\left(=0.124 m_{0}\right)$ for the electron effective mass of ZnTe, $m_{e}^{* \prime}\left(=0.15 m_{0}\right)$ for the electron effective mass of $\mathrm{ZnSe}, m_{h}^{*}\left(=0.64 m_{0}\right)$ for the heavy hole effective mass of $\mathrm{ZnTe}, m_{h}^{* \prime}\left(=0.8 m_{0}\right)$ for the heavy hole effective mass of $\mathrm{ZnSe}$.

Because sufficiently large numbers of component functions (see Eq. (A9) in Appendix A) must be taken to ensure that the eigenenergies approach their asymptotic values, we begin our discussion with the determination of the matrix dimension $N$. We choose the radius of the outer cylinder $\rho_{0}$ to be $400 \AA$ and the height of the outer cylinder $H$ to be $150 \AA$ (see Fig. A1). By examining how fast the wave functions or the probability densities converge, so long as they converge in a range where they are much smaller than the inter dot distance, $417 \AA$, measured from AFM as mentioned earlier, our choice of the outer cylinder size $\left(\rho_{0}\right.$ and $\left.H\right)$ is justified. We discuss the convergence of the probability densities later. Set $h_{0}=11 \AA, D=275 \AA(\rho=0.04)$ and arbitrarily choose $q=2$. The ground state energies of the heavy hole (electron), $E_{H}\left(E_{e}\right)$, confined by a lens shaped $\mathrm{ZnTe} \mathrm{QD}$ (by the infinite barriers) as a function of the matrix dimension $N$ are obtained by solving the matrix equation and are presented in Fig. 7. Here, we see that when $N$ is 900 , the ground state energies $E_{H}$ converge to a value of $256.77 \mathrm{meV}$ and the variation of $E_{e}$ with $N$ is slow $\left(13.75<E_{e}<13.82\right)$. To ensure the convergence of the confinement energies, we choose $N=900$ in the following calculations.

Although the barrier height $\Delta E_{c}^{0}$ and the well depth $\Delta E_{h}^{0}$ of a QD is not known, the value of $q$ can be determined by examining the PL spectrum. On the one hand, we observed that the PL spectrum peaked around $E_{\mathrm{op}}=2.185 \mathrm{eV}$ and the line shape can be well described by a Gaussian with a mean of $\langle E\rangle=2.185 \mathrm{eV}$ and a variance of $\sigma^{2}=0.002 \mathrm{eV}^{2}$ :

$$
I=7500 \exp \left[-\left(E_{\mathrm{op}}-\langle E\rangle\right)^{2} / 2 \sigma^{2}\right],
$$

as shown by the dotted curve in Fig. 2. Here, $E_{\mathrm{op}}$ is the optical energy. On the other hand, we knew that on average the dot height distribution peaked around $h_{0}=25 \AA$ as indicated in Fig. 6. Recall that the offset of $h_{0}$ is $14 \AA$. To search a value of $q$ such that $E_{\mathrm{op}}=E_{e H}$ (see Eq. (1)) is located at $2185 \mathrm{meV}$ with $h_{0}=25-14=11(\AA)$ and $D=275 \AA(\alpha=0.04)$, we solved a $900 \times 900$ matrix for the sub-band energies as a function of $q$, and plotted the result in Fig. 8. Unexpectedly, we find that when q ranges from 0.6 to 1.4, $E_{\mathrm{op}}$ as a function of q is linear:

$$
E_{\mathrm{op}}(\mathrm{meV})=-377.5 q+2592.9 .
$$

Thus, for $E_{\mathrm{op}}=2185 \mathrm{meV}$, a roughly approximated value of $q$ is 1.1 and yields band edge discontinuities of $\Delta E_{c}^{0}=315 \mathrm{meV}$ (barrier) for the conduction band and $\Delta E_{v}^{0}=735 \mathrm{meV}$ (well) for the valence band. 


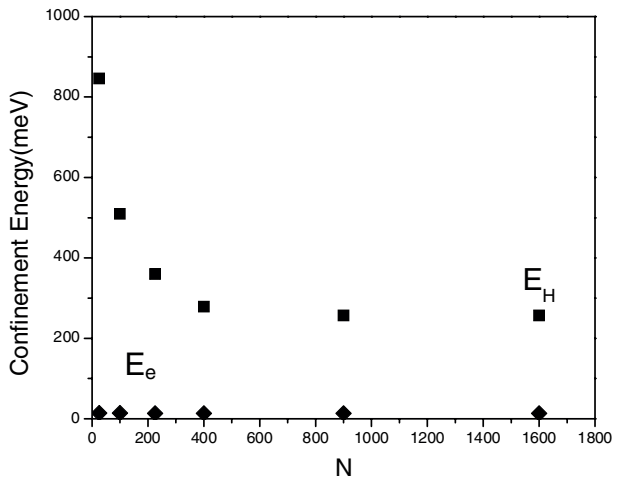

Fig. 7 Convergence of the ground state confinement energies for the heavy hole $E_{H}$ and for the electron $E_{e}$ as a function of the matrix dimension $N$ with $q=2, h_{0}=24 \AA$ and $D=275 \AA$.

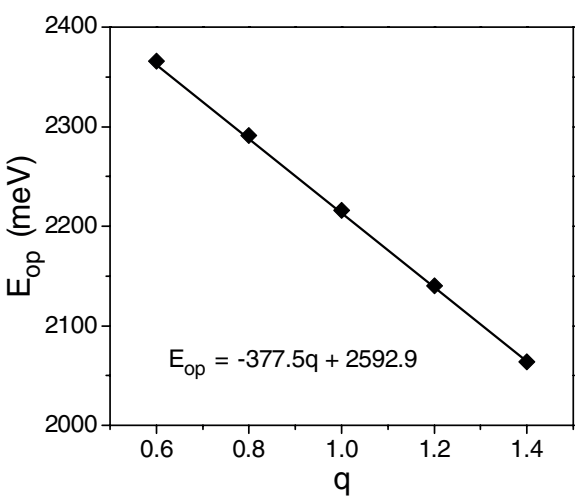

Fig. 8 Variation of the optical energy $E_{\mathrm{op}}$ with $q$ for $h_{0}=24 \AA$ and $D=275 \AA$.

When $q$ is 1.1 , the ground state energies are calculated to be $E_{e}=13.6 \mathrm{meV}, E_{H}=226.7 \mathrm{meV}$ and the corresponding probability densities are plotted in Fig. 9. The wave functions are normalized in the atomic units as mentioned in APPENDIX A. Refer to Figs. A1 and A2 for a lens shaped QD and recall that lens height $h_{\text {lens }}$ decreases as $\mathrm{r}$ increases (see Eq. (A16)). The variations of the ground state probability density as a function of $z$ with $r=0,60,80$ and $100 \AA$ for the electron are presented in Fig. 9(a) and with $r=0,24,32$ and $40 \AA$ for the heavy-hole are presented in Fig. 9(b). Inside the type II ZnTe/ZnSe QDs, electrons see a barrier $\left(\Delta E_{c}^{0}\right)$ while holes see a well $\left(\Delta E_{v}^{0}\right)$; therefore, we expect that most of the electrons must reside outside the QDs as indicated in Fig. 9(a) and most of the holes must reside inside the QDs as indicated in Fig. 9(b). Figure 9(a) shows that when $r$ is 0 and $0 \leq z \leq h_{\text {lenz }}=h_{0}=11 \AA$ (inside QD), the probability density possesses a dip (a local minimum) located at $z=7 \AA$. Although most electrons reside in the matrix material, the region without $\mathrm{QD}(z<0)$ is populated more. These results become even more evident when $r$ increases and $h_{\text {lenz }}$ decreases. When $r$ is 60, 80 and $100 \AA$, the dip which shifts towards the $z$-axis locates at $z=5,4$ and $3 \AA$, and the corresponding probability density increases rapidly which means that most electrons prefer to reside outside the QD. When $r$ is greater than the lens base radius $(D / 2=137.5 \AA$ ), the dip in the probability density diminishes (not shown in Fig. 9). Fi-
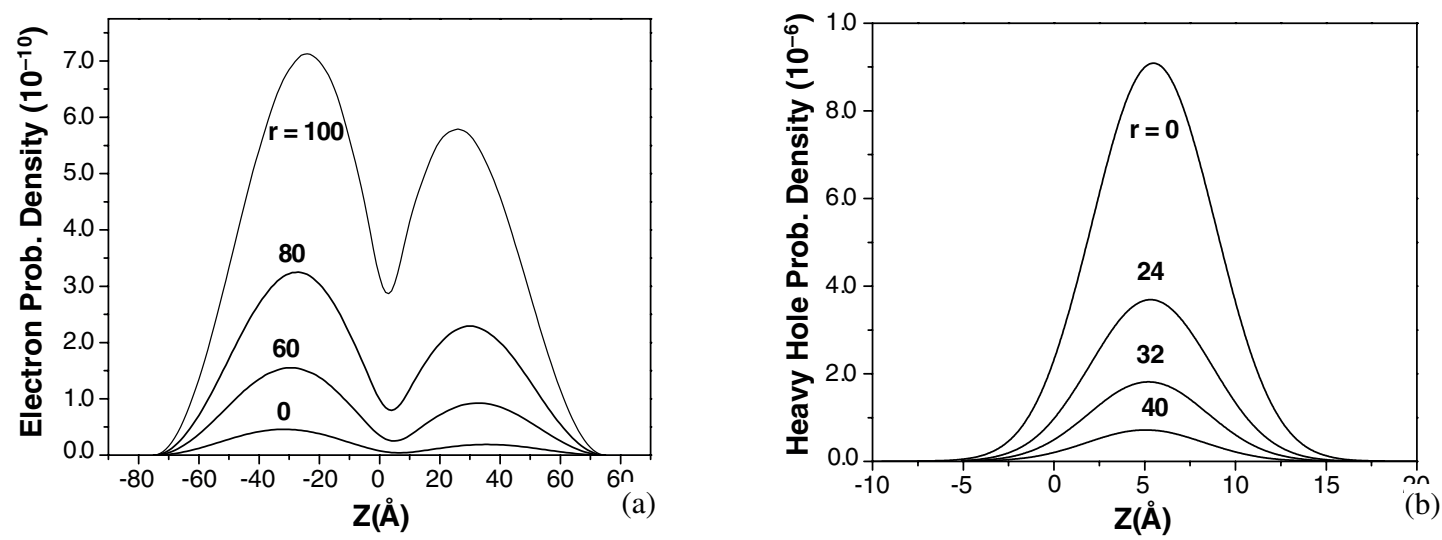

Fig. 9 Variation of the probability densities as a function of $z$ with $r$ located at (a) $r=0,60,80$ and $100 \AA$ for the conduction electron and (b) $r=0,24,32$ and $40 \AA$ for the heavy hole. 


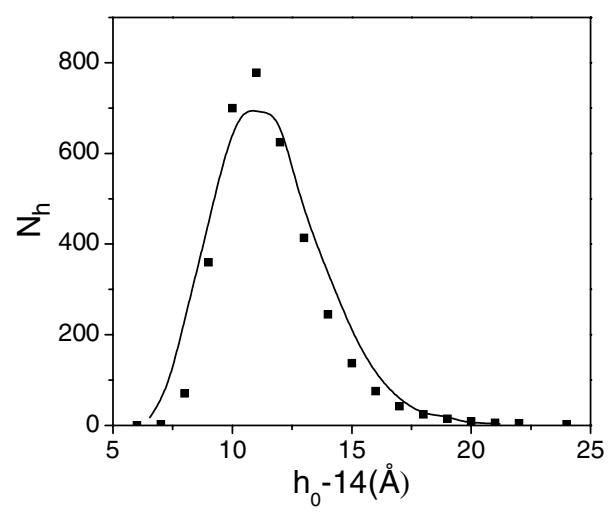

Fig. 10 Comparison of $N_{h}$ versus $h_{0}-14$ obtained from the AFM measurement as shown in Fig. 5 (solid curve) and the PL spectrum as shown in Fig. 2 (squares).

gure 9(b) shows that when $r$ increases from 0 to $40 \AA$, the probability density rapidly decreases and most holes are localized inside the QD $(0 \leq z \leq .11 \AA)$. Although the outer cylinder was arbitrarily set to have a large size of $\rho_{0}=400 \AA$ and $H=150 \AA$, the probability densities converge within a range of $\Delta z=25 \AA$ $(\ll H)$ in the $z$-direction and $\Delta r=60 \AA\left(\ll \rho_{0}\right)$ in the $r$-direction. Therefore, the choice of the size of the outer cylinder is justified. Recall that the inter dot distance is $417 \AA$ which is much larger than $\Delta z$ and $\Delta r$, thus our earlier assumption that there are no carrier communications between dots holds.

To infer the dot size distribution from the spectrum, we calculate the ground sub-band energies as a function of $\mathrm{h}_{0}$ for both the conduction electron and the hole with $q=1.1, D=h_{0} / \alpha, \alpha=0.04, H=150 \AA$ and $\rho_{0}=400 \AA$. For each value of $h_{0}$, we solve the matrix equation of Eq. (A10) to obtain a corresponding value of $E_{\mathrm{op}}=E_{e H}$ from Eq. (1). Using Eq. (3), we convert the PL spectrum ( $I$ as a function of $\left.E_{\mathrm{op}}\right)$ as shown in Fig. 2 to a relation of $\mathrm{N}_{\mathrm{QD}}=\mathrm{N}_{h}$ as a function of $\mathrm{h}_{0}$ and is marked in Fig. 10 with squares. Although the PL line shape is a Gaussian, the dot size distribution $\mathrm{N}_{h}$ is a deformed Gaussian because the eigenenergies obtained from the Schrödinger equation as a function of $h_{0}$ are definitely non-linear. By shifting a $14 \AA$ offset in $h_{0}$, AFM measured data as shown in Fig. 5 is plotted in Fig. 10 with a solid curve. Here, we see that the agreement between data obtained from the PL (squares) and the AFM (solid curve) as shown in Fig. 10 are excellent. Therefore, we conclude that the result of the PL spectrum ( $I$ versus $E_{\mathrm{op}}$ ) can be interpreted as the result of the AFM measurement $\left(\mathrm{N}_{\mathrm{QD}}\right.$ versus dot size) via the Schrödinger equation.

\section{Summary}

We have studied the PL spectrum, which indicates that the ZnTe/ZnSe QD structure belongs to a type II band alignment. Besides the main features of the ZnSe buffer layer located at $2.820 \mathrm{eV}$, a broader structure peaking at $2.185 \mathrm{eV}$ was observed in the PL spectrum at $10 \mathrm{~K}$. The broadness of the PL represents the spatial inhomogeneity of the QD sizes. The PL spectrum was determined to be a normal distribution with a variance of $0.002 \mathrm{eV}^{2}$. However, the dot size distribution is a deformed Gaussian because the energy as a function of the dot size obtained from the Schrödinger equation is definitely non-linear. Based on only one set of data of dot size distribution or sample measured by AFM, statistical estimates with standard errors were evaluated using the bootstrap method. Means, percentiles and correlation coefficients for the base diameter and the height of the QDs were used to infer the probability distributions. We concluded that the dot distributions were indeed a deformed Gaussian distribution and the correlation coefficient of the diameter and the height of the QDs was $0.49 \pm 0.01$. The uniformity of the dot size distribution was poor. In order to infer the dot size distribution from the PL spectrum, we numerically solved the Schrödinger equation in the OPF approximation for the type II lens shaped QDs. The convergence of the probability density was discussed in a great detail. Our analyses concluded that the band edge discontinuities inside the dot is a barrier with $\Delta E_{c}^{0}=315 \mathrm{meV}$ for the conduction band and is a well with $\Delta E_{v}^{0}=735 \mathrm{meV}$ for the valence band. In the AFM measurement, the offset in dot height is $14 \AA$ and the aspect ratio is 0.04 . By converting the PL spectrum ( $I$ versus $\left.E_{\mathrm{op}}\right)$ to the AFM data $\left(N_{\mathrm{QD}}\right.$ versus dot 
size) through the Schrödinger equation, the agreement between these two sets of data was excellent. Therefore, we conclude that the result of the PL spectrum can be interpreted as the result of the AFM measurement.

Acknowledgements This work was supported by the National Science Council of Taiwan, R.O.C., under the grant numbers of NSC-92-2112-M-009-041 and NSC-91-2112-M-033-005.

\section{Appendix: \\ OPF approximation for the type I and II symmetroidal QDs}

Consider a large cylinder of a barrier material with radius $\rho_{0}$ and height $H$ that surrounds a QD of a well material with the shape of the symmetroid determined by rotating the curve $z=f(r)$ on the $r-z$ plane about the $z$ axis, as shown in Fig. A1. Suppose that a particle with an effective mass $m^{*}(r, z)$ is confined by a finite potential barrier $V(r, z)$ because of the shape of the dot in the cylindrical coordinates $(r, \varphi, z)$. We take the band edge of the well region as the energy reference. Therefore, if the particle is inside the QD (well) region, we assign $m^{*}(r, z)=m_{w}$ and $V(r, z)=0$; otherwise we assign $m^{*}(r, z)=m_{b}$, $V(r, z)=V_{0}$ and on the outer boundary we have $V(r, z)=\infty$. The subscripts $w$ and $b$ in $m_{w}$ and $m_{b}$ refer to the well and the barrier, respectively. For convenience, we adopt the Rydberg Ry $=13.56 \mathrm{eV}$ as the unit for the energies and the Bohr radius $r_{B}=0.53 \AA$ as the unit for the lengths. The free electron mass is $m_{e}$. By using these units, the three-dimensional time-independent Schrödinger equation for the envelope function in the effective mass approximation becomes dimensionless and is given by:

$$
-\left(\nabla \frac{m_{e}}{m^{*}(r, z)} \cdot \nabla\right) \Psi(r, \varphi, z)+V(r, z) \Psi(r, \varphi, z)=E \Psi(r, \varphi, z)
$$

and

$$
\nabla=\hat{e}_{r} \frac{\partial}{\partial r}+\hat{e}_{\varphi} \frac{1}{r} \frac{\partial}{\partial \varphi}+\hat{e}_{z} \frac{\partial}{\partial z}
$$

where $E$ and $\Psi$ are the eigen-energy and the eigen-function, respectively. Eqs. (A1) and (A2) ensure that the Hamiltonian operator is Hermitian and the current probability density is continuous even for a spacedependent effective mass $m^{*}(r, z)$ at the interface of the heterojunction.

The envelope function of a QD, $\Psi(r, \varphi, z)$, is then expanded in terms of a set of OPF, $\psi_{n m l}(r, \varphi, z)$, where quantum numbers $n, m$ and $l$ are positive integers. Suppose that in Fig. 1 the QD is as large as the cylinder and the effective mass $m^{*}$ is $m_{e}$. Then $\psi_{n m l}(r, \varphi, z)$ must satisfy the Helmholtz's equation, a cylindrical problem with infinite barrier height,

$$
\nabla^{2} \psi_{n m l}+K^{2} \psi_{n m l}=0
$$

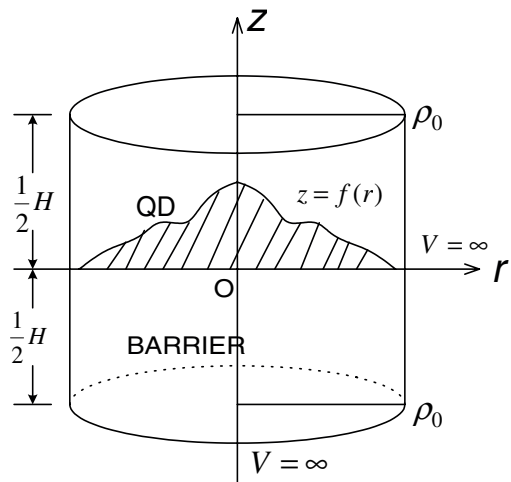

Fig. A1 Coordinates and symbols of a symmetroidal quantum dot (QD) surrounded by a cylindrical barrier material. 
with the following boundary conditions: $\psi_{n m l}=0$ at $r=\rho_{0}$ or $z= \pm H / 2$. The OPF, $\psi_{n m l}(r, \varphi, z)$, are given by:

$$
\begin{aligned}
& \psi_{n m l}(r, \psi, z)=R_{n m}(r) Z_{1}(z) \Phi_{m}(\varphi), \\
& \Phi_{m}(\varphi)=\frac{1}{\sqrt{2 \pi}} \exp (i m \varphi), \quad 0 \leq \varphi \leq 2 \pi, \\
& R_{n m}(r)=\frac{\sqrt{2}}{\rho_{0} J_{m+1}\left(k_{n} \rho_{0}\right)} J_{m}\left(k_{n} r\right), \quad 0 \leq r \leq \rho_{0}, \\
& Z_{1}(z)=\sqrt{\frac{2}{H}} \sin l \pi\left(\frac{1}{2}-\frac{z}{H}\right), \quad-H / 2 \leq z \leq H / 2,
\end{aligned}
$$

where $R_{n m}, Z_{1}$ and $\Phi_{m}$ are orthonormal functions. The boundary conditions acquire a relation

$$
K^{2}=k_{n}^{2}+\left(\frac{l \pi}{H}\right)^{2}
$$

where $J_{m}(x)$ is the Bessel function of order $\mathrm{m}$ and $k_{n} \rho_{0}$ is the $n$-th root of $J_{m}\left(k_{n} r\right)$ at $r=\rho_{0}$ (i.e., $\left.J_{m}\left(k_{n} \rho_{0}\right)=0\right)$. The envelope function is finally expressed as a series by adopting $\psi_{n m l}(r, \varphi, z)$ as bases with appropriately chosen coefficients as follows:

$$
\Psi(r, \varphi, z)=\sum_{n, m, l} B_{n m l} \psi_{n m l}(r, \varphi, z),
$$

where $B_{n m l}$ are the linear coefficients.

Substituting expression (A9) into Eq. (A1), multiplying the left side by $\psi_{n^{\prime} m^{\prime}}^{*}$, and finally integrating over the volume of the cylinder yields the matrix equation

$$
A_{n m l n^{\prime} m^{\prime} l^{\prime}}-E \delta_{n n^{\prime}} \delta_{m m^{\prime}} \delta_{l l^{\prime}}=0 .
$$

The matrix elements are given by [19, 22-24]

$$
A_{n m l n^{\prime} m^{\prime} l^{\prime}}=\iiint_{\text {cylinder }} \mathrm{d} \Omega\left[\frac{m_{e}}{m^{*}} \nabla \psi_{n^{\prime} m^{\prime} l^{\prime}}^{*} \cdot \nabla \psi_{\mathrm{nml}}+\psi_{n^{\prime} m^{\prime} l^{\prime}}^{*} V \psi_{n m l}\right] .
$$

where the element of volume in cylindrical coordinates is $\mathrm{d} \Omega=r \mathrm{~d} r \mathrm{~d} \varphi \mathrm{d} z$. We split the integral into three parts, within each of which the effective mass is constant: first, we take an integral with $m^{*}=m_{b}$ over the whole cylinder (i.e., barrier plus QD well regions); second, we subtract the integral with $m^{*}=m_{b}$ over the QD well region; and third, we add the integral with $m^{*}=m_{w}$ over the QD well region. Taking advantage of the azimuthal symmetry of the QDs, the triple integrals in Eq. (A11) can be simplified to be the single integrals

$$
\begin{aligned}
& \iiint_{\mathrm{QD}} \mathrm{d} \Omega \nabla \psi_{n^{\prime} m^{\prime} l^{\prime}}^{*} \cdot \nabla \psi_{n m l}=\int_{0}^{\rho} \mathrm{d} r\left[r \frac{\mathrm{d} R_{n^{\prime} m}^{*}}{\mathrm{~d} r} \frac{\mathrm{d} R_{n m}}{\mathrm{~d} r} F_{l^{\prime} l}(z(r))+\frac{m^{2}}{r} R_{n^{\prime} m}^{*} R_{n m} F_{l^{\prime} l}(z(r))\right. \\
& \left.+\frac{\pi^{2} l^{\prime} l}{H^{2}} r R_{n^{\prime} m}^{*} R_{n m} G_{l^{\prime} l}(z(r))\right] \delta_{n^{\prime} m}, \\
& \iiint_{\mathrm{QD}} \mathrm{d} \Omega \psi_{n^{\prime} m^{\prime} l}^{*} \psi_{n m l}=\int_{0}^{\rho} \mathrm{d} r r R_{n^{\prime} m}^{*} R_{n m} F_{l^{\prime} l}(z(r)) \delta_{m^{\prime} m}, \\
& F_{l^{\prime} l}(z(r))=\frac{2}{H} \int_{0}^{z} \mathrm{~d} z \sin l^{\prime} \pi\left(\frac{1}{2}-\frac{z}{H}\right) \sin l \pi\left(\frac{1}{2}-\frac{z}{H}\right),
\end{aligned}
$$




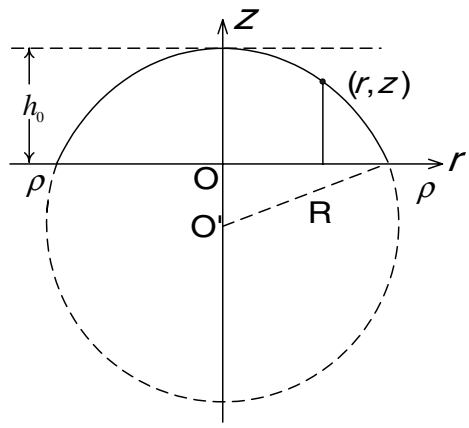

Fig. A2 Coordinates and symbols for QDs in the shape of a lens.

$$
G_{l^{\prime} l}(z(r))=\frac{2}{H} \int_{0}^{z} \mathrm{~d} z \cos l^{\prime} \pi\left(\frac{1}{2}-\frac{z}{H}\right) \cos l \pi\left(\frac{1}{2}-\frac{z}{H}\right) .
$$

The matrix elements can be evaluated numerically as long as the shape or profile of a QD, i.e. $z=f(r)$, is defined. A schematic picture of a lens-shaped QD as a part of a sphere of radius $R$ is shown in Fig. A2. For convenience, let's take the center of the lens base $O$ as a reference. The lens has a height $h_{0}$ and a base radius $\rho$. A relation between $z$ and $r$ can be obtained from the geometry of the shape:

$$
z(r)=h_{\text {lens }}=\sqrt{R^{2}-r^{2}}-\sqrt{R^{2}-\rho^{2}} \geq 0, \quad r \leq \rho .
$$

Substituting Eq. (A16) into Eqs. (A12-A15) together with Eq. (A11), the matrix elements for a lensshaped type I QD are determined.

For the type II structure, we take the material with the lower energy band edge as the energy reference, hence we have to replace $V$ as $-V$ in Eq. (A1). Accordingly, the matrix elements in Eq. (A11) are modified as

$$
A_{n m l n^{\prime} m^{\prime} l^{\prime}}=\iiint_{\text {cylinder }} \mathrm{d} \Omega\left[\frac{m_{e}}{m^{*}} \nabla \psi_{n^{\prime} m^{\prime} l^{\prime}}^{*} \nabla \psi_{n m l}-\psi_{n^{\prime} m^{\prime} l^{\prime}}^{*} V \psi_{n m l}\right] .
$$

\section{References}

[1] P. M. Petroff, A. C. Gossard, R. A. Logan, and W. Wiegmann, Appl. Phys. Lett. 41, 635 (1982).

[2] F. E. Prins, G. Lehr, M. Burkard, H. Schweizer, M. H. Pilkuhn, and G. W. Smith, Appl. Phys. Lett. 62, 1365 (1993).

[3] M. A. Reed, R. T. Bate, K. Bradshaw, W. M. Duncan, W. M. Frensley, J. W. Lee, and H. D. Smith, J. Vac. Sci. Technol. B 4, 358 (1986).

[4] P. M. Petroff and S. P. Denbaars, Superlattices Microstruct. 15, 5 (1994).

[5] Y. Nabetani, T. Ishikawa, S. Noda, and A. Sakai, J. Appl. Phys. 76, 347 (1994).

[6] J. Y. Marzin, J. M. Gérard, A. Izraël, D. Barrier, and G. Bastard, Phys. Rev. Lett. 73, 716 (1994).

[7] F. M. Peeters and A. Schweigert, Phys. Rev. B 53, 1468 (1996).

[8] R. Notzel et al., Appl. Phys. Lett. 66, 2525 (1995).

[9] J. Y. Marzin and G. Bastard, Solid State Commun. 92, 437 (1994).

[10] S. Fafard, R. Leon, D. Leonard, J. L. Merz, and P. M. Petroff, Phys. Rev. B 52, 5752 (1995).

[11] B. Damilano, N. Grandjean, F. Semond, J. Massies, and M. Leroux, Appl. Phys. Lett. 75, 962 (1999).

[12] K. Kitamura, H. Umeya, A. Jia, M. Shimotomai, Y. Kato, M. Kobayashi, A. Yoshikawa, and K. Takahashi, J. Cryst. Growth 214/215, 680 (2000).

[13] I. Daruka, J. Tersoff, and A.-L. Barabási, Phys. Rev. Lett. 82, 2753 (1999).

[14] S. Lee, I. Daruka, C. S. Kim, A.-L. Barabási, J. L. Merz, and J. K. Furdyna, Phys. Rev. Lett. 81, 3479 (1998).

[15] Y. Wu, K. Arai, and T. Yao, Phys. Rev. B 53, R10485 (1996). 
[16] M. C. Harris Liao, T. H. Chang, Y. F. Chen, J. W. Hsu, J. M. Lin, and W. C. Chou, Appl. Phys. Lett. 70, 2256 (1997).

[17] T. Tawara, S. Tanaka, H. Kumano, and I. Suemune, Appl. Phys. Lett. 75, 235 (1997).

[18] Johnson Lee, W. C. Chou, and G. J. Jan, Appl. Phys. Lett. 81, 2082 (2002).

[19] S. Gangopadhyay and B. R. Nag, Nanotechnology 8, 14 (1997).

[20] D. Gershoni, H. Temkin, G. J. Dolan, J. Dunsmuir, S. N. G. Chu, and M. B. Panish, Appl. Phys. Lett. 53, 995 (1988).

[21] J. Shertzer and L. R. Ram-Mohan, Phys. Rev. B 41, 9994 (1990).

[22] M. Califano and P. Harrison, J. Appl. Phys. 88, 5870 (2000).

[23] M. Califano and P. Harrison, J. Appl. Phys. 86, 5054 (2000).

[24] M. Califano and P. Harrison, Phys. Rev. B 61, 10959 (2000).

[25] A. Wojs, P. Hawrylak, S. Fafard, and L. Jacak, Phys. Rev. B 54, 5604 (1996).

[26] M. A. Cusack, P. R. Briddon, and M. Jaros, Phys. Rev. B 54, R2300 (1996).

[27] M. Grundmann, O. Stier, and D. Bimberg, Phys. Rev. B 52, 11969 (1995).

[28] O. Stier, M. Grundmann, and D. Bimberg, Phys. Rev. B 59, 5688 (1999).

[29] A. J. Williamson and A. Zunger, Phys. Rev. B 59, 15819 (1999).

[30] G. Liu and S. L. Chuang, J. Appl. Phys. 88, 5554 (2000).

[31] B. Efron and R. J. Tibshirani, An Introduction to the Bootstrap, Monographs on Statistics and Applied Probability, Vol. 57 (Chapman \& Hall/CRC Press, 1993).

[32] A. C. Davison and D. A. Hinkley, Bootstrap Methods and their Application, Cambridge Series in Statistical Probabilistic Mathematics (Cambridge University Press, 1997).

[33] Y. Homma, H. Yamaguchi, and Y. Horikoshi, Appl. Phys. Lett. 68, 63 (1996).

[34] J. C. Kim, H. Rho, L. M. Smith, H. E. Jackson, S. Lee, M. Dobrowolska, and J. K. Furdyna, Appl. Phys. Lett. 75, 214 (1999).

[35] J. C. Kim, H. Rho, L. M. Smith, H. E. Jackson, S. Lee, M. Dobrowolska, J. L. Merz, and J. K. Furdyna, Appl. Phys. Lett. 73, 3399 (1998).

[36] E. H. Lee, S. Stoltz, H. C. Chang, M. H. Na, H. Lou, and A. Petrou, Solid State Commun. 107, 177 (1998).

[37] O. Madelung, Numerical Data and Functional Relationships in Science and Technology, Vol. 22: Semiconductors, Subvolume a: Intrinsic Properties of Group IV Elements and III-V, II-VI and I-VII Compounds (Springer-Verlag, Berlin, 1987). 